\title{
CENNI SULLE FORME DELLA CONTAMINAZIONE DAL NOVECENTO A OGGI
}

\author{
Nota del s.c. CLELIA MARTIGNONI (*) con LORENZO DONGHI (**), \\ ELISA ENRILE $(* * *)$, GIORGIA GHERSI $(* * * *)$
}

(Adunanza del 6 febbraio 2020)

SuNTO. - Il saggio si concentra su alcuni aspetti culturali-chiave del Novecento e dell'età contemporanea nel campo delle scienze umane. In particolare analizza la specifica "contaminazione" realizzatasi costantemente tra più stili, nel rapporto tra le arti, tra $\mathrm{i}$ generi, i linguaggi, le forme espressive, che frequentemente coinvolgono l'interdisciplinarità. Gli autori illustrano alcuni tra i molti esempi di "contaminazione" nel campo di cinema, arte, letteratura, anche discutendone in breve il significato culturale.

$* * *$

Abstract. - Notes on "Contamination" in the Twentieth Century and Beyond.

The essay focuses on some key cultural features of the twentieth century and of the contemporary world as related to humanistic knowledge. In particular, it deals with the specific aspect of "contamination" across multiple styles as arts, genres, languages and expressive forms, which frequently involve "interdisciplinarity". The authors illustrate some of the many examples of "contamination", especially in the fields of cinema, art and literature, also by briefly discussing its cultural significance.

(*) Università degli Studi di Pavia, Istituto Lombardo. Accademia di Scienze e Lettere, Milano, Italy. E-mail: clelia.martignoni@unipv.it

(**) Università degli Studi di Pavia, Italy. E-mail: lorenzo.donghi@unipv.it

${ }^{(m+k)}$ Fondazione Remo Bianco, Milano, Italy. E-mail: elisa.enrile1@gmail.com

(*mik) Università di Pisa, Italy. E-mail: giorgia.ghersi@phd.unipi.it 


\section{IN PREMESSA}

\section{Clelia Martignoni}

Veloce, complesso, inquieto, il Novecento ha attraversato con energia e spirito inventivo e innovativo nei saperi umanistici moltissime avventure culturali e disparate stagioni; e, come a tutti è noto, rivoluzionari avanzamenti hanno interessato gli studi scientifici e i settori tecnici e della comunicazione, con vere e proprie svolte epocali che hanno determinato potenti mutamenti generali. Diciamo Novecento per riferirci a fenomeni culturali nati spesso tempestosamente (è il caso delle avanguardie storiche primonovecentesche e poi delle neo-avanguardie) nel XXI secolo, ma che si proiettano oltre e perdurano nei decenni che stiamo vivendo, tra evoluzioni e cambiamenti anche imprevedibili, sempre all'insegna di quella velocità che è un carattere costituivo del secolo alle nostre spalle, su cui infatti abbiamo aperto.

Per dirla con lo storico della contemporaneità Giovanni De Luna, una «folla di definizioni [...] si è abbattuta sul secolo appena finito» (De Luna scriveva queste parole nel 2004), catturando in sintesi incisive le singolarità novecentesche. Non è inutile ricordarle qui pur di corsa per mettere in luce la generale problematicità con cui gli storici e molti degli stessi protagonisti hanno percepito il Novecento. Pensiamo alla notissima denominazione di «secolo breve» che si deve a Eric Hobsbawm, che ne ha proposto addirittura una nuova scansione cronologica: 1914-1991, racchiuso cioè tra due eventi di massimo rilievo storico-politico, la Grande Guerra e la caduta del muro di Berlino con la fine dell'Unione Sovietica. Ma è pure notevole che Hobsbawm abbia dato all'edizione originaria inglese del suo libro, 1994, il titolo significativo Age of Extremes, e che nella traduzione italiana (1995) del Secolo breve compaia il sottotitolo non meno lampante L'età dei grandi cataclismi. Il filosofo britannico Isaiah Berlin, nato in Lettonia ed ebreo, tutti dati ben influenti sulla sua esperienza biografica e ideologica, ha parlato del Novecento come del «secolo più terribile della storia occidentale»; lo scrittore francese Jean-Marie Domenach, del «secolo dell'orrore inaudito e dell'utopia senza misura» (1995). Sulla stessa linea, lo storico Marco Revelli ha definito il Novecento «secolo degli opposti» (2001); e il filosofo Salvatore Natoli secolo di «progresso e catastrofe» (1999) ${ }^{1}$. 
Una pur breve selezione di questi molteplici tentativi di classificare i caratteri più notevoli dell'inquieto secolo ce ne restituisce con forza un'immagine carica di opposizioni e contrasti , sospesa com'è tra gli eccellenti avanzamenti scientifici e tecnici e gli inauditi orrori sperimentati dalla condizione umana. Mentre - come tutti ricordano data la fortuna dell'immagine - l'epoca postmoderna, che intervenne dagli anni settanta, è stata denominata «liquida» dal sociologo Zygmunt Bauman, grazie alle incertezze e destabilizzazioni collettive e personali (la perdita del senso dello stato, il tramonto di ideologie e istituzioni, il trionfo del soggettivismo e del consumismo) che possono essere vissute sia in negativo sia in positivo.

I contrasti che dominano il secolo e il sentimento ancora odierno della liquidità ci introducono nella questione che qui cercheremo di accennare, insieme con un drappello di allievi e quasi allievi dell'Università di Pavia che ho il piacere di introdurre e di accompagnare nei loro interventi: Lorenzo Donghi, Elisa Enrile, Giorgia Ghersi, (l'ordine è alfabetico), giovanissimi ricercatori e dottorandi formatisi all'Università di Pavia, che si stanno specializzando rispettivamente in storia e critica del cinema, arte contemporanea e letteratura contemporanea.

Nella nostra nota il tema prescelto su cui ci siamo confrontati in più occasioni e a più riprese nel tempo è infatti spiccatamente novecentesco e oltre (nel senso suddetto che esso abita pleno jure anche que-

discussioni e controversie, cfr. almeno M. Revelli, Oltre il Novecento, Torino, Einaudi, 2001; M. Salvati, Il Novecento. Intepretazioni e bilanci, Roma-Bari, Laterza, 2001; P. Sorcinelli (a cura di), Identikit del Novecento. Conflitti, trasformazioni sociali, stili di vita, Roma, Donzelli, 2004; G. De Luna, La passione e la ragione, Milano, Bruno Mondadori, 2004; C. Pavone (a cura di),'900. I tempi della storia, Roma, Donzelli, 1998. Molto ricco e pieno di interesse il bilancio multidisciplinare uscito per Laterza (1996) curato e introdotto con incisività da Corrado Stajano La cultura italiana del Novecento (che ha conquistato una fama nazionale inopinata essendo Stajano un intellettuale stabilmente controcorrente perché nelle prove di maturità del 2019 è stato proposto un brano dell'introduzione per commentarlo).

2 Altri contrasti molto forti si rilevano nelle pagine introduttive di Corrado Stajano appena cit., dove vengono riportati a mo' di avvio istruttivo al volume i giudizi di autorevoli protagonisti dei più svariati settori.

3 Rinviamo ai suoi studi fondamentali: Liquid modernity, Cambridge (UK) 2000 (trad. it. di S. Minucci, Modernità liquida, Roma-Bari, Laterza, 2002), e Liquid love, ivi, 2003 (trad. it. di S. Minucci, Amore liquido, ivi, 2004). 
sti primissimi decenni del nuovo millennio e che è destinato a ulteriori metamorfosi ed evoluzioni), e ne rappresenta bene le oscillazioni e il gusto per mescolanze ed equilibri precari e incerti: la contaminazione o l'ibridismo tra più linguaggi ed espressioni artistiche, non sempre di segno progressivo, ottimistico, "corretto", talora anzi produttivo di visioni beffarde e trasgressive $e^{4}$. Ciò mi richiama un'epigrafe estrema, a valenza negativa, che desidero citare anche per l'esplicita carica ideologica oppositiva: voluta per una loro retrospettiva del 2012 (Milano, Hangar Bicocca) da due grandi artisti visivi e cineasti, forti sperimentatori, Yervant Gianikian e Angela Ricci Lucchi (lui armeno, lei emiliana, scomparsa nel 2108). Radicale anche il loro lavoro artistico, che si basa sul recupero e sul rimontaggio creativo di spezzoni di vecchi filmati, sul filo di storie collettive e di potenti drammi del Novecento (colonialismo, migrazioni, esilio). Nella mostra, provocatoria sin dal titolo Non non non, spiccava questa sintesi drammatica del contemporaneo: «Non pulito, non estetico, non educativo, non progressivo, non cooperativo, non etico, non coerente: contemporaneo».

Aggiungo un altro aspetto-chiave della contemporaneità e già della modernità novecentesca: la ricerca interdisciplinare, punto di forza nei gruppi delle avanguardie storiche, che hanno sempre lavorato all'incrocio tra più arti, letteratura, teatro, cinema, fondendo e intrecciando linguaggi e forme espressive.

La contaminazione e la mescolanza degli stili hanno trionfato poi su piani più estesi e allargati nel pieno Novecento, in coincidenza con l'avvento della nuova comunicazione telematica, negli anni segnati dall'affermarsi del Postmoderno, che ha prodotto un profondo mutamento di tutti gli assetti culturali, e che si è posto all'inizio (decennio Settanta del Novecento) come un insieme di tendenze al massimo "inclusive", eclettiche, citazioniste, ironiche e raffinate. Moltissimi i campi coinvolti: diciamo in primis architettura e urbanistica perché hanno avuto un ruolo trainante: basti citare l'indagine di Robert Venturi del 1972 in elogio di Las Vegas, contro l'alto-modernismo architettonico, accusato di elitarismo e

4 Sento il bisogno di precisare, per l'eventuale lettura da parte di filologi, che non è assolutamente in campo la nozione specialistica di «contaminazione» centrale invece nella filologia del testo e studiata dai maggiori filologi del Novecento (Pasquali, Contini, Segre, Avalle, ecc.), cioè dell'assunzione di lezioni appartenenti a rami diversi della tradizione. E naturalmente va da sé che il termine è impiegato in senso specifico in campo biologico, fisico, chimico. 
conservatorismo5. Ma poi citiamo l'arte, la letteratura, il teatro, il cinema, la fotografia, la musica, l'estetica, la filosofia, l'antropologia, l'intero complesso delle scienze umane. Infranti i confini tra categorie tradizionali (come cultura alta di élite/cultura di massa e popolare, individualità/pluralità, maschile/femminile, animale/umano), contaminazione e ibridazio$n \mathrm{e}^{6}$ sono diventate idee-chiave novecentesche. Ne sono via via derivate anche nel sentire comune una serie di percezioni collettive, così schematizzabili secondo i numerosi studi sull'argomento: restringimento dello spazio, perdita della profondità storica, moltiplicazione e frammentazione delle esperienze, dentro un quadro del tutto inedito di globalizzazione e planetarizzazione.

Interdisciplinarità e transdisciplinarità hanno interessato alcune grandi dottrine novecentesche, come la complessità, che ha genesi scientifiche, di ambito matematico-fisiche (si potrebbe fissare un punto di partenza nei celebri studi di fine Ottocento del matematico e fisico teorico francese Henri Poincaré), con interferenze multiple con l'“effetto-farfalla" individuato dallo statunitense Edward Lorenz nei primi anni sessanta, con le teorie del Caos, con quella dei sistemi complessi, con la cibernetica e naturalmente un'influenza profonda è stata provocata dal decisivo avvento dei computer. Ma la complessità conosce anche uno sviluppo di grande fascino che interessa gli studi sociologici, filosofici ed epistemologici di Edgar Morin, dagli anni settanta-ottanta in avanti, anche con intense applicazioni pedagogiche. È qui che Morin articola come cardine sostanziale della complessità il riferimento interdisciplinare e transdisciplinare, a favore di una generale "riforma del pensiero" felicemente trasferibile nell'insegnamento ${ }^{7}$.

5 Cfr. R. Venturi, D. Scott Brown, S. Izenour, Learning from Las Vegas, Cambridge (USA), MIT Press, 1972. Prima trad. italiana: Imparare da Las Vegas, Venezia, Cluva, 1985; ora: M. Orazi (a cura di), Macerata, Quodlibet, 2010.

6 Sono numerosi gli studiosi che preferiscono parlare, anziché di «contaminazione», di «ibridazione» (termine di origine biologico-genetica), o di «ibridismo», insistendo sull'atipicità morfologica. Cfr. in Italia E. Fiorani, J. Ceresoli, Ibridazioni: nuovi territori della scienza e della tecnica, dell'arte, della mente, Milano, Apèiron, 2000 (e più in generale: E. FIORANI, Panorami del contemporaneo, Milano, Lupetti, 2009). In ambito architettonico-urbanistico (di cui si è già sottolineato il particolare spicco) si veda il catalogo di J. Fenton, Hybrid Buildings, New York-San Francisco, 1985, dedicato al contesto nord-americano. Ricchissima la bibliografia: si veda almeno: A. F. Per, J. Mozas, J. Arpa, This is bybrid. An analysis of mixed-use buildings, Vitoria, a+t Ediciones, 2011.

7 Nella ricchissima produzione di Morin, su questi aspetti specifici si vedano: 
Un'altra prospettiva novecentesca di grande interesse che si giova dell'interdisciplinarità è offerta dagli Studi visuali, o Visual Studies, o Visual Culture, sviluppatisi negli anni ottanta-novanta e irradiatisi dagli ambienti universitari anglosassoni. Gli Studi visuali si richiamano a molti precedenti culturali europei (da Benjamin, da Foucault al recupero di Warburg a Barthes) e hanno imposto ciò che i teorici maggiori hanno chiamato «Pictorial turn» (John Mitchell, 1992) o «Iconic turn» (Gottfried Boehm, 1994) sul modello del «Linguistic turn» (Richard Rorty, 1967) per segnalare una nuova decisiva svolta epistemologica grazie alla prospettiva visuale insediatasi al posto di quella pan-linguistica già predominante. I Visual Studies hanno reimpostato radicalmente la valutazione e interpretazione critica delle immagini, lavorando soprattutto sul legame con istituzioni, contesti e apparati socio-culturali, considerando sia i soggetti che producono le immagini e il contesto d'origine, sia i soggetti e i contesti che le recepiscono, osservano, consumano, trasmettono. L'ambito è dunque particolarmente vasto e investe più discipline correlate: oltre all'arte, filosofia ed estetica, storia e storia sociale, storia delle idee, antropologia, ecc., e i media e i new media visivi e digitali. Nicholas Mirzoeff (nato nel 1962), docente di Media, Culture and Communications all'Università di New York, con An introduction to visual culture (1999; trad. it. Introduzione alla cultura visuale, 2005), e con How to See the World (2015, trad. it. Come vedere il mondo, 2017), sposta l'attenzione dall'arte verso la centralità dell'immagine nella vita collettiva e individuale, potenziata dagli strumenti di Internet e della rete globale, e arriva a sviluppare una storia e un "pensiero" delle immagini tra il XX e il XXI secolo, insistendo sulla prevalenza del cosiddetto "attivismo visuale".

Mi piace chiudere citando in questi dintorni Antinomie, recentissimo blog collettivo-rivista italiano online (https://antinomie.it/) che si intitola non a caso al principio del contrasto e che è stato fondato nel 2020 da Andrea Cortellessa, Federico Ferrari e Riccardo Venturi. Scrivono i tre fondatori: «Finalità di Antinomie è tenere traccia delle

Une tête bien fait. Repenser la réforme, réformer la pensée, 1999, trad. it. La testa ben fatta. Riforma dell'insegnamento e riforma del pensiero, Milano, Raffaello Cortina, 2000; e Les Sept Savoirs nécessaires à l'éducation du futur, 2000; trad. it. I sette saperi necessari all'educazione del futuro, ivi, 2001; Enseigner à vivre. Manifeste pour changer l'éducation, 2014 , trad. It. Insegnare a vivere. Manifesto per cambiare l'educazione, a cura di Mauro Ceruti, ivi, 2015. 
forme di scrittura (poesia, letteratura, saggistica, filosofia, critica, ecc.) che, attraverso il superamento dei confini disciplinari, hanno posto al cuore della propria pratica il rapporto tra la parola e le immagini». Di Federico Ferrari, che insegna Filosofia dell'arte e Fenomenologia delle arti contemporanee all'Accademia di Belle Arti di Brera, citiamo dal testo a tre introduttivo, Ouverture, I gennaio 2020, queste parole che disegnano nuove realtà culturali in fieri, immerse nella complessità, nell'interdisciplinarità, nella contaminazione:

Ogni anno, il genere umano produce più di un trilione di immagini. Un trilione significa un miliardo di miliardi, 1000000000000000 000. È un numero che non può essere davvero pensato da una mente umana. Se consideriamo, infatti, che in epoca medievale un uomo entrava in contatto, nell'arco della sua intera esistenza, con circa quaranta immagini artificiali - mentre oggi si calcola che siano circa dodici miliardi abbiamo la misura di un passaggio epocale senza precedenti. Pare, quindi, del tutto pertinente affermare che la nostra civiltà stia vivendo la transizione da una biosfera fondata sulla materia a un'iconosfera, in cui la vita tende a smaterializzarsi nell'impalpabilità dell'immagine. Un'epoca, quella dell'iconosfera, che potremmo definire come l'epoca dell'immagine del mondo, cioè l'epoca in cui $[\ldots]$ il mondo stesso diviene un'immagine.

Il futuro come si diceva, all'interno della precarietà o liquidità generale che si è accennata, potenziata nei mesi in cui scriviamo dalla crisi profonda generata dalla pandemia globale del Covid 19, è imprevedibile, ma alcuni aspetti cui si è qui accennato sembrerebbero difficilmente reversibili.

\section{NOVECENTO E OLTRE: IL RILIEVO DELLA CONTAMINAZIONE}

\section{Lorenzo Donghi}

Tra le molteplici definizioni con cui abbiamo archiviato il secolo che ci siamo lasciati alle spalle, ma in cui affondano le radici alcuni fenomeni, cruciali e inesausti, che ancora segnano il tempo che abitiamo, una in particolare assume uno spiccato rilievo: il Novecento è stato il secolo della contaminazione. Un concetto, quest'ultimo, non certo inedito né ovviamente assente prima, ma che, appunto nel XX secolo, ha vissuto una stagione di costante esaltazione. 
Constatazione forse perentoria, ma in fondo riscontrabile in ogni ambito e settore. Del resto, dall'accelerazione vertiginosa dei trasporti alle repentine trasformazioni occorse al mondo delle comunicazioni, dalla libera circolazione di merci e di persone provocata dai processi di globalizzazione alla condivisione su scala mondiale di idee, voghe e problematiche, dal melting pot che fonda il tessuto sociale delle metropoli più popolose, delineando una condizione di continua esposizione della propria identità al confronto con l'altro, fino ai fenomeni di ibridismo culturale e linguistico che ne sono conseguiti, si può infatti ben dire che quello passato sia stato il secolo che ha fatto della contaminazione il suo motore, e al contempo il suo irrinunciabile criterio guida.

La considerazione è valida - ed è quel che più ci pertiene - anche per tutto ciò che è avvenuto nel quadro dell'intero sistema delle arti e delle lettere, se è vero che il Novecento ha conferito al contaminare non solo lo spessore di un precipuo modus operandi, bensì la valenza del suo più ambizioso progetto estetico. Anche il recente volume $A$ mezzi termini. Forme della contaminazione dal XX secolo del 2019 rappresenta un'altra tra le numerose occasioni di confronto e riflessione aperte sul tema ${ }^{8}$.

Vale anzitutto la pena indugiare un poco sul termine chiamato in causa, fortemente ricorsivo anche in accezioni metaforiche e allusive, tutte però allineate nel configurare un peculiare modo di procedere del pensiero e dell'azione. Già la sua ricostruzione etimologica offre d'altronde spunti indicativi. La parola contaminare, di origine incerta quanto di nascita antica, sconta infatti una derivazione dal verbo latino tangere, comune anche alle voci contatto e contagio. Due riferimenti significativi, la cui convocazione permette di cogliere appieno la duplicità semantica insita alla contaminazione: fenomeno che viene così a definirsi non solo come condizione di vicinanza tra elementi distinti, ma che, giocoforza e non facoltativamente, prescrive una qualche interazione tra gli stessi.

Una prossimità dunque foriera di sconfinamenti, che predica la per-

8 Cfr. L. Donghi, E. Enrile, G. Ghersi (a cura di), A mezzi termini. Forme della contaminazione dal XX secolo, Milano, Mimesis, 2019, con scritti di Paolo Giovannetti, Pietro Benzoni, Niccolò Scaffai, Giuliano Cenati, Giuliana Benvenuti, Roberto Pinto, Elio Grazioli, Paolo Campiglio e Lorenzo Donghi. Saggio introduttivo di Clelia Martignoni. Il volume raccoglie gli interventi del Convegno organizzato da G. Ghersi ed E. Enrile presso il "Collegio Nuovo - Fondazione Sandra ed Enea Mattei", Università di Pavia, 12 aprile 2018. 
dita di qualsiasi fisionomia riconoscibile, che incoraggia la profanazione di ogni forma pura, e che incentiva la fondazione di un'identità rinnovata, basata sui capisaldi dell'incrocio, della mescolanza e della con-fusione. Contaminazione, pertanto, come processo attivo e dinamico, che può essere di incontro e sodalizio come di scontro e di frizione, ma sotto la cui egida, in ogni caso, si registrano fenomeni di influenza, trasmissione o scambio. Ebbene, di una tendenza tanto reiterata, che percorre con moto obliquo il corso di tutto il Novecento, due sono i momenti topici e salienti, interpretabili come espressioni di altrettanti contesti che coincidono, rispettivamente, con l'alba e il tramonto del secolo breve.

Il primo è ovviamente la stagione delle avanguardie storiche: quel «brontolio sotterraneo», secondo la calzante formula di Vasilij Kandinskij' , che agita l'orizzonte artistico europeo nei primi due decenni, facendosi sintomo di un rinnovamento estetico radicale. Con l'intento di rompere ogni legame con la tradizione, e animati dall'urgenza di superare la dominanza di istanze realistiche, figurative e mimetiche, i movimenti d'avanguardia, pur spesso differendo in premesse ed esiti, esasperano infatti all'unisono l'importanza della libertà espressiva, svincolando ogni manifestazione artistica da retaggi ereditati o canoni prestabiliti. Tra le arti crollano così i perimetri, i confini si trasformano in frontiere mobili, e l'idea di specificità viene meno, o ne diventa superflua l'individuazione: pittori, musicisti, registi, fotografi, architetti e artisti di ogni sorta si ritrovano a condividere progetti comuni, la cui vivace impertinenza mira consapevolmente a destabilizzare lo spettatore, abituato fin lì a confrontarsi con opere chiare e inequivocabili.

A sua volta, difatti, l'opera d'arte perde quei caratteri di unicità, inscindibilità e interna coerenza che le avevano conferito a lungo lo statuto di un tutto organico. Assumendo i tratti di una struttura complessa e composita, eterogenea, articolata in frammenti, messa letteralmente insieme a partire da una manovra di aggregazione che pone in dialogo elementi originariamente estranei, spesso peraltro difformi e disparati, che trovano tuttavia un'inedita e reciproca collocazione quando a farsene carico è il gesto del montaggio ${ }^{10}$. Principio certo molto generale, e che in quanto tale risulta applicabile a diversi linguaggi, tecniche e

9 V. Kandinskij, Alcune nozioni sull'arte sintetica, in Id., Tutti gli scritti, vol. II, Milano, Feltrinelli, 1981, 199 (or. 1927).

10 Restituire in questa sede la straordinaria mole di interventi che inquadrano 
materiali, ma che, non a caso, suscita un'attrazione irresistibile anzitutto nei confronti del cinema: quella «settima arte» ${ }^{11}$, scrive Ricciotto Canudo, che si impone immediatamente come forma esemplare della contaminazione, capace di racchiudere e conciliare le altre discipline, ben più longeve, e di sostenere un autentico rilancio dell'ideale wagneriano dell'opera d'arte totale.

Il secondo momento combacia invece con il profilarsi della condizione postmoderna ${ }^{12}$, che nell'ultimo quarto di secolo irrompe con veemenza nel quadro delle società occidentali, influenzandone drasticamente il dibattito accademico e impattandone tutti i livelli della produzione artistica. In contrasto con lo spirito utopico e avanguardista tipico dell'ideologia modernista, e caratterizzandosi anzitutto in quanto portavoce di

il principio del montaggio sullo sfondo del primo Novecento non potrebbe che risultare un proposito arrischiato, viziato peraltro da un inesorabile carattere di inesaustività. Basti ricordare che, oltre alle sperimentazioni artistiche riconducibili agli approcci di numerosi artisti d'avanguardia (tra cui László Moholy-Nagy, Fernand Léger, Marcel Duchamp, Man Ray, Francis Picabia, e molti altri), il montaggio diventa assoluto protagonista anche nel quadro di alcune fondamentali proposte teoriche, rispetto cui i primi decenni del secolo scorso svolgono la funzione di roboante cassa di risonanza. Tra esse - e solo per fare alcuni esempi - si annoverano il filone iconologico inaugurato da Aby Warburg, concretizzatosi nell'organizzazione di un vero e proprio "atlante delle immagini" (Bilderatlas Mnemosyne); la concezione storiografica discontinuista di Walter Benjamin, elaborata in aperto contrasto con la tradizione storicista; la produzione filmica di Sergej Michajlovic Ejzenstejn, così come del resto quella saggistica, sofisticatissima e più volte rimaneggiata; la ricerca visiva condotta da Georges Bataille sulle pagine della rivista «Documents» (1929-1930).

11 R. Canudo, Manifeste des Sept Arts, «7 arts», 23 novembre 1922. Con evidente richiamo alla distinzione lessinghiana tra arti figurative e poesia, Canudo, considerato il primo teorico ad aver riflettuto sul cinema in modo sistematico, elegge quest'ultimo a sintesi delle arti tradizionali, muovendo dal presupposto che le due arti fondamentali siano la musica (da cui derivano le arti ritmiche come poesia e danza) e l'architettura (che ha invece generato le arti plastiche, come pittura e scultura). Va però ricordato come Canudo, un decennio prima, avesse già parlato del cinematografo come della sesta arte, nel cui spazio d'azione l'autore rintraccia, sulla scorta dell'estetica hegeliana, cinque diversi generi artistici: musica, poesia, architettura, pittura e scultura. Cfr. Id., La nascita di una sesta arte, in «Cinema Nuovo», n. 225, settembre-ottobre 1973, 361-371 (or. 1911).

$12 \mathrm{Si}$ fa evidentemente riferimento a F. Lyotard, La condizione postmoderna. Rapporto sul sapere, Milano, Feltrinelli, 1981 (or. 1979). Per una trattazione ampia e onnicomprensiva, tra le più complete guide orientative alla temperie postmoderna citerei tuttora P. Carravetta, Del postmoderno. Critica e cultura in America all'alba del Duemila, Milano, Bompiani, 2009. 
una profonda e generale crisi, lo Zeitgeist postmoderno reifica la sua smaliziata assiologia a partire da un novero di presupposti inderogabili: tra essi, la delegittimazione delle metanarrative illuministe, quali l'emancipazione universale e l'idea di progresso; una concezione disincantata della storia, deprivata di ogni andamento teleologicamente orientato e, almeno nelle formulazioni più catastrofiste, giunta niente meno che al suo termine; la liquidazione di ogni verità che si proponga come maiuscola e condivisa, sostituita, in prospettiva relativista, da una pluralità di discorsi che rivendicano una validità puntuale, circoscritta e contingente.

Nel milieu postmoderno, la contaminazione diventa dunque il principale strumento operativo di una rinnovata sensibilità che, in contrasto con le criticità messe in evidenza, assume paradossalmente un carattere euforico, rutilante, e come noto primariamente ironico. Abolite le differenze tra cultura "alta" e cultura "bassa", in nome della definitiva affermazione di una produzione orizzontale, popolare, massificata, e assunto un atteggiamento spregiudicato nel saccheggiare archivi e repertori del passato, sono infatti forme e modelli del citazionismo ad assumere la dignità di impareggiabile modalità espressiva. Il dialogo intertestuale, l'ibridazione stilistica e il pastiche, così come la prassi artistica del riuso e del reimpiego, costituiscono infatti la linfa che nutre quell'eclettismo, in cui si accumulano appropriazioni arbitrarie e non raramente indebite, che contribuisce al successo di una concezione estetica squisitamente postmoderna.

Un approccio, va sottolineato, favorito anche da fattori strettamente tecnologici. Si pensi infatti ai cambiamenti registrati, a cavallo tra i due secoli, all'interno di un complesso delle comunicazioni condizionato prima dalla multimedialità (cioè dalla compresenza interattiva di più codici, linguaggi e supporti che si risolve nel ricorso simultaneo a differenti media), e poi dalla definitiva affermazione del digitale (ossia di un sistema di codifica delle informazioni che, condiviso da tutti i dispositivi, elabora segnali discreti per rappresentare segnali continui sotto forma di valori numerici). Media e tecnologie hanno infatti scandito le tappe di un percorso che, sebbene in costante aggiornamento, è giunto oggi a piena maturazione: una rincorsa che ha interessato tutte le forme della comunicazione, coinvolgendo primariamente le arti e sospingendole a ridosso di ulteriori livelli della contaminazione. Fenomeno, quest'ultimo, che dopo essere stato incubato per tutto il Novecento, ha così saputo consolidarsi anche nei primi decenni del nuovo secolo, fino a reclamare il suo valore di provata matrice del contemporaneo. 
2. L'ARTE, LE ARTI

\section{Elisa Enrile}

Se fino a inizio Novecento 1' "Arte" era concepita solo nell'esito grafico riversato su tela, legno o intonaco, oppure plasmato e ricavato da marmo o ceramica, con l'inizio del nuovo secolo qualcosa cambia completamente. Come è già stato detto nelle pagine che precedono il mio breve intervento, con le avanguardie storiche prima e con le neoavanguardie poi, fino agli esiti propriamente contemporanei, il significato e il concetto di "prodotto" artistico si estende sempre di più, perde ogni nettezza nei contorni e si apre a nuove tecniche e modalità, che generano nuovi significati. Una contaminazione continua e prolungata, che tocca discipline e materie diverse per ricrearsi sempre nuova, con aspetti talora familiari, talora decisamente perturbanti. In un momento culturale e storico in cui risulta difficile dire cosa è e cosa non è degno di essere considerato "Arte", risulta più semplice e immediato definire cosa lo è stato e quali nuove forme hanno via via acquisito dignità e interesse presso il pubblico e la critica.

Uno dei risultati più comuni e riconosciuti è quello ottenuto dall'incontro tra parola scritta e immagine, connubio che non si declina solo nella vicinanza o mescolanza di testo e figura, ma anche nella comunione di intenti tra le due pratiche, nella volontà di narrarsi a vicenda, nel tentativo degli scrittori di raccontare gli artisti, e degli artisti di rappresentare gli scrittori.

Considerando l'arte post-duchampiana il momento di svolta per un naturale avvicinamento tra questi due territori culturali, è possibile individuare anche un consistente numero di scrittori e narratori che hanno sconfinato con la loro produzione in entrambi i campi: tra questi si possono ricordare Filippo de Pisis, pittore e autore di saggi e poesie; Dino Buzzati, scrittore e pittore; o ancora Alberto Savinio e Carlo Levi ${ }^{13}$. Ma oltre a questa casistica, è interessante tenere presente quel fenomeno che porta alla collaborazione tra i professionisti afferenti a entrambi i campi e che restituisce esiti di duplice lettura molto interessanti. A partire dalla

13 Rimando per un quadro generale al saggio di V. Bramanti, Artisti scrittori, Bergamo, Lubrina editore, 1988. 
fertile amicizia tra Leonardo Sciascia e Ferdinando Scianna, che spesso permette al primo di dare ancora più forza e vigore alle proprie parole grazie alle espressive fotografie del secondo ${ }^{14}$. Interessante anche il caso dell'artista francese Sophie Calle e degli scrittori Paul Auster e Enrique Vila-Matas, o ancora il rapporto di quest'ultimo con l'artista Dominique Gonzalez-Foerster: un sapiente gioco di rimandi dove gli scrittori si trovano a essere soggetti di opere e le artiste personaggi di romanzi ${ }^{15}$. Il Novecento, oltre che aprire un nuovo amplissimo ventaglio di possibilità in ambito culturale, ha anche il merito di dare dignità ad alcuni mezzi che fino a quel momento non erano considerati come attivatori di "prodotti" artistici. Primo tra tutti appunto la fotografia, nata nella prima metà dell'Ottocento e usata per molto tempo semplicemente per catturare nel modo più veritiero possibile il paesaggio e l'architettura, e poi per ritrarre la borghesia e il popolo. Lentamente la macchina fotografica ha smesso di essere considerata solamente uno strumento "neutrale" per guadagnare invece lo statuto e il valore riconosciuto di produttore di contenuti inediti. A partire dalla seconda metà dell'Ottocento la fotografia si allontana dai binari percorsi fino a quel momento, per divenire con l'avvento del nuovo secolo medium di numerose sperimentazioni: prima accreditata come strumento utile per l'informazione, con il passare degli anni viene impiegata in ambito pubblicitario, commerciale, di moda, ma anche senz'altro estetico-artistico. Gli esempi sono molteplici: l'americano David LaChapelle, molto attivo nel campo della moda e della pubblicità, cerca di lasciare il segno rendendo ogni suo scatto un'opera di pop art dai colori cangianti e dai soggetti inediti. Interessante anche il lavoro di altri fotografi che si muovono sul labile confine tra fotoreportistica e foto d'arte, come per esempio il celebre Franco Fontana, che utilizza il colore e la forte geometrizzazione per rendere ogni paesaggio molto più di una semplice "ripresa da finestrino"16.

14 Cfr, alcune opere come L. Sciascia, Feste religiose in Sicilia. Fotografia di Fernando Scianna, Roma, Casa editrice Leonardo da Vinci, 1965. Molto fitta la bibliografia su questi aspetti: in particolare cfr. sul rapporto di Sciascia con Scianna ma anche su altri temi collegati (la relazione tra letteratura e le arti visive, le collaborazioni con il cinema): Maria Rizzarelli, Sorpreso a pensare per immagini. Sciascia e le arti visive, Pisa, ETS, 2013.

15 R. Pinto, Incroci tra arte e letteratura, in A mezzi termini, op. cit.,123 - 141.

16 Rimando per un quadro generale al testo: S. Heller, J. Heimann, Fotografia dal XX secolo, Colonia, Taschen, 2012. Per un approfondimento su David 
Proprio riferendoci al campo della moda, e guardando alla città di Milano, capitale indiscussa del settore, emblematico risulta essere il caso di Davide Mosconi. L'artista non si limita solo a riportare figure umane in abiti di classe, ma gioca con la superficie incidendola e trasfigurandola, in un intricato gioco di collage e asportazioni di pellicola che sfocia anche in dittici monocromatici o quasi astratti che giocano sul concetto di tridimensionalità ${ }^{17}$.

Nonostante al giorno d'oggi si tenda a volte ad andare controcorrente, abbandonando i vezzi del cinema hollywoodiano per far emergere l'importanza del realismo del materiale e delle scenografie, con una fedeltà quasi sacrale alla riproduzione di fonti documentarie e iconografiche $^{18}$, il cinema è un campo in cui la tentazione della sperimentazione è stata sempre molto presente.

Dopo la macchina fotografica infatti, un altro mezzo che acquista forza e si rimodella è la macchina da presa, protagonista del Novecento, che dalle sperimentazioni del regista sovietico Dziga Vertov con il suo discusso e originalissimo L'uomo con la macchina da presa (1929) in avanti, realizza appieno tutta la sua spinta innovatrice e le sue potenzialità narrative. La contaminazione tra arte e "ripresa" risulta evidente nella pratica rivoluzionaria del montaggio, un procedimento fondamentale dal punto di vista tecnico, strutturale, inventivo, e altrettanto efficace per sperimentare diversi modi di sovrapporre immagini e diverse prospettive di rappresentare. Il montaggio infatti non si limita a raccontare una storia, anzi è quell'azione che permette di aumentare la complessità della storia che si accinge a raccontare. Interessante è il caso di un regista come Harun Farocki, film maker tedesco che concentra la sua ricerca sul rimontare immagini già esistenti per la creazione di prodotti originali, con un senso

LaChappelle: http://home.davidlachapelle.com/; per un approfondimento su Franco Fontana: http://francofontanaphotographer.com/.

17 E. Grazioli, Il corpo dell' immagine, in A mezzi termini, op. cit., 143-152.

18 Si veda ad esempio lo studio del linguista e critico stilistico Pietro Benzoni, il quale porta l'attenzione proprio su questa pratica, analizzando accuratamente il caso de Il mestiere delle armi (2001) di Ermanno Olmi, tratto da Li ultimi fatti d'arme dello Ill.mo Sig.r Joanni da le Bande Nere, riportato anche nel sottotitolo a rimarcare immediatamente l'intenzione, non molto comune nella storia dell'italiano da pellicola, di restituire quasi filologicamente l'epoca in questione, non solo tramite le ambientazioni e i costumi, ma anche attraverso le scelte linguistiche. Cfr. P. Benzoni, Tra nuda cronaca e sacra rappresentazione in A mezzi termini, op. cit., 39-63. 
e un significato altro da quello di partenza, come nel caso dell'opera Videogramme einer Revolution, in cui l'autore mette al centro la rivoluzione che avvenne in Romania nel dicembre dell's9 nel tentativo di ricostruire i fatti accaduti tramite le immagini che li rappresentano ${ }^{19}$. Ma Farocki non è ovviamente il solo a usare il montaggio come occasione sperimentale. Interessante citare un regista esemplare come Luis Buñuel, che qualche anno prima di lui aveva già giocato con il montaggio con l'artista Salvador Dalì, facendo del "taglio" l'elemento centrale del suo celebre Un chien andalou (1929), fortemente assimilabile ad un grande quadro realista in movimento. O ancora Jean Luc Godard, che più o meno negli stessi anni di Buñuel ha tentato quasi di abbattere la quarta parete inserendo nei suoi film un forte tentativo di interazione tra narratore e spettatore $^{20}$. Nel discorso complessivo di contaminazione culturale, è imprescindibile accennare alla nuova forma artistica che nasce intorno alla metà del secolo e che mette al centro dell'opera la componente corporale e relazionale dell'artista con il pubblico: l'arte performativa. Partendo dal gruppo Fluxus, network interdisciplinare e internazionale nato in America USA negli anni Sessanta, fino ai giorni nostri, gli artisti che hanno fatto dell' "happening" la propria firma autoriale sono molti. Cito almeno Alan Kaprov, che con il suo 18 bappening in 6 parts del '59 ha fatto da apripista per il fertile movimento dell'arte performativa. Così come il caso dell'azionismo viennese con Hermann Nitsch, dove la forte corporalità e carnalità si unisce alla sacralità artistica in performance dall'alto valore misterico ${ }^{21}$. Una delle performer sicuramente più acclamate è Marina Abramović, nella cui produzione tematiche ancestrali si fondono agli istinti più umani. Che lavorasse da sola o con il compagno di una vita Ulay ${ }^{22}$, i concetti portati sotto i riflettori dall'artista jugoslava

19 L. Donghi, Contaminazione come montaggio. Il cinema, l'opera di Harun Farocki e il caso di Videogramme einer Revolution , in A mezzi termini, op. cit., 159-181.

20 Cfr. almeno su questi aspetti D. Tomasi, Lezioni di regia. Modelli e forme della messinscena cinematografica, Torino. UTET, 2004

21 E. Grazioli (a cura di), Arte dal 1900. Modernismo, antimodernismo, postmodernismo, Bologna, Zanichelli, 2017.

22 Ulay, compagno storico della Abramović, è morto a marzo 2020 dopo aver combattuto a lungo contro il cancro. Artista, fotografo e accademico tedesco, è considerato una delle figure centrali dell'arte performativa del secolo scorso. Dal sodalizio amoroso e lavorativo con l'artista serba sono nate alcune delle performance più iconiche della modernità come "The Wall Walk in China" (1988), dove i due scelgono di 
sono sempre stati potenti e d'impatto, così come l'atto realizzativo finale. In lotta contro gli stereotipi di genere e le ipocrisie della società contemporanea, l'artista si serve di una vasta simbologia quasi sacrale per ribellarsi, usando il suo corpo come tramite tra il pubblico e il suo percepito ${ }^{23}$.

\section{LETTERATURA DI PAROLE, IMMAGINI, SPAZI}

\section{Giorgia Ghersi}

Se la cultura occidentale a partire da Platone ha accusato le forme e le poetiche dell'ibridazione letteraria di corrompere la purezza originaria dell'arte favorendone la confusione estetica e la degenerazione, è tra Settecento e Ottocento, e poi pienamente dal Novecento, che il concetto di "contaminazione" va incontro a una traslazione positiva ${ }^{24}$ e che le arti, e tra esse anche la letteratura, iniziano a subire, per dirla con Giovanni Bottiroli, il fascino della cosiddetta «ideologia dell'ibrido» ${ }^{25}$. Si afferma dunque l'idea secondo la quale un'opera tanto più mescola strategie stilistiche, discorsive e formali tipiche di diversi generi e media, tanto più acquista una patente di sperimentalismo e interesse agli occhi almeno di alcuni studiosi. La natura tutt'oggi non pacificata della categoria di "ibrido" emerge nell'idea conflittuale che viene associata ai prodotti che sotto questa etichetta vengono classificati: un rapporto di forze, uno scontro che vede alternativamente vincitore l'uno o l'altro dei generi di partenza individuati nel testo, in un gioco che, nei suoi esiti più sterili, si limita a sottolineare la divergenza dalle forme pure originali più che il

percorrere a piedi la muraglia cinese partendo dai due capi opposti per ritrovarsi al centro in un abbraccio che sancisce la loro separazione.

23 La bibliografia sull'artista è ovviamente molto vasta. Per un quadro generale: M. Abramović, J. Kaplan, Attraversare i muri. Un'autobiografia, Milano, Bompiani, 2018; J. Westcott, Quando Marina Abramović morirà, Monza, Johan \& Levi editore, 2011; P. Campiglio, Nostalgia del sacro nella performance di Marina Abramović, in A mezzi termini, op. cit., 153-157.

24 Cfr. per un'analisi del tema a largo respiro M. Douglas, Purezza e pericolo. Un'analisi dei concetti di contaminazione e tabù, Bologna, Il Mulino, 1975; N. García Canclini, Culture ibride. Strategie per entrare e uscire dalla modernità, Milano, Guerini e Associati, 1998; P. Burke, Cultural Hybridity, Cambridge, Polity, 2009.

25 G. Bottiroli, Ibridare, problema per artisti. Alcune tesi, in «Enthymema» I (2010), 155. 
rinnovato vigore che quelle stesse assumono in un panorama socio-culturale fortemente rinnovato. In questo senso è con acutezza che Paolo Giovannetti, nel volume citato A mezzi termini, suggerisce che «in ballo non c'è solo la fisionomia dei singoli partecipanti all'ibrido: ma che decisivo è il punto di partenza, la direzionalità, la vettorialità della mescolanza: c'è un punto, cioè un genere, di riferimento che muove verso un altro genere (o altri generi); invertendo la freccia del movimento, la sostanza dell'operazione cambia» ${ }^{26}$. Accantonando le accuse di "sterilità" ${ }^{27}$ e cercando di tenersi parimenti lontani dalla "moda dell'ibridismo" che attribuisce alla contemporaneità l'esclusiva di una tecnica che a ben vedere caratterizza la grande letteratura fin dalle sue origini, è innegabile che «la critica letteraria de[bba] oggi fare i conti con il fatto che le distinzioni nette, a cominciare da quelle tra romanzo (erede inquieto dell'epica), poesia e teatro, sono inadeguate, e forme come il saggio e la vasta galassia della non-fiction sembrano rispondere meglio a nuove necessità espressive ${ }^{28}$. Questa prospettiva, che programmaticamente si oppone alle codificazioni rigide, ai confini e alle barriere non solo tra i generi ma anche tra le varie forme artistiche, è stata ampiamente sperimentata nei linguaggi del Novecento, e ha trovato nuovo terreno fertile alla fine del secolo e alle soglie del nuovo millennio sotto l'influenza di Internet e dei social $^{29}$, in una cultura sempre più "liquida" e aperta alla transmedialità

26 P. Giovannetti, Prosa d'arte, prosa ritmica, poesia in prosa (per non parlare della prosa in prosa). Questioni storiche e di metodo, in A mezzi termini, op. cit., 24. Si tratta di un movimento bidirezionale esemplificato bene dai diversi effetti della mescolanza tra prosa e poesia nei suoi vari esiti dal poemetto baudelairiano, al frammento vociano che esaspera la sonorità del dettato, fino ai più recenti esperimenti di "prosa in prosa" e prosa ritmica rap caratterizzati da una «poetica dello scarto, del residuo, dell'oggetto povero, "trovato" o "ritrovato", ed esibito fuori di ogni ambizione estetizzante».

27 Sempre secondo Bottiroli vanno sotto l'etichetta di "cattivo ibridismo" o "ibridismo sterile" quelle opere in cui le forze in gioco nella mescolanza, anziché stimolarsi a vicenda, si ostacolano e si paralizzano. L'idea che un'opera ibrida, in quanto organismo originale, sia sterile cioè non riproducibile, è già di Lessing. Cfr. G. E. Lessing, Drammaturgia d'Amburgo, a cura di P. Chiarini, Roma, Bulzoni, 1975.

28 È questo l'assunto da cui partono Paolo Gervasi, Francesca Lorandini e Pietro Taravacci nella premessa al numero monografico di «Ticontre» che, sotto la loro cura, si propone di riflettere sull'ibridazione dei generi come caratteristica peculiare nella creazione artistica dal Romanticismo alla contemporaneità. Cfr. «Ticontre. Teoria Testo Traduzione» 5 (2016), Mash-up. Forme e valenze dell'ibridazione nella creazione letteraria.

29 A questo proposito si tenga presente, per esempio, A. Antoniazzi, Labirinti 
in cui la contaminazione si propone come modalità-chiave dell'esperienza artistica.

È nell'ottica di un'erosione dei confini tra discipline che si colloca anche ad esempio (tra i moltissimi che si potrebbero scegliere) un'inversione di tendenza tra il campo di pertinenza della letteratura e quello del giornalismo, sempre più punto di partenza piuttosto che di arrivo. Sono molti gli scrittori oggi della modernità e della contemporaneità che sono entrati ed entrano nel campo letterario in quanto giornalisti o reporter e che portano nei loro libri tecniche e tempi della scrittura di inchiesta, complicati da un ulteriore compromesso tra il verbale e il visivo. Il reportage, forma tipicamente legata all'atto di descrizione, talora di denuncia di un autore-reporter che viaggia nei luoghi di interesse per riportare ciò che ha visto e sentito, si lega nel graphic journalism al fumetto che, a partire dal tardo Novecento, si accosta alla realtà con intento conoscitivo-testimoniale. Gli interessanti esempi di Joe Sacco, Guy Delisle, Aleksandar Zograf, Igort e Zerocalcare richiamano l'attenzione sulla sfasatura temporale su cui si basa questa tipologia testuale che fonde l'intento della restituzione immediata della notizia e una tecnica dal tempo lungo come il disegno di strisce fumettistiche. Forme e modi della narrazione fumettistica ne escono rimodulati in funzione di un giornalismo di approfondimento che segna in maniera nuova i tempi della comunicazione informativa.

La varietà di forme e contaminazioni che il fumetto innesca con tipologie discorsive quali il reportage giornalistico e il romanzo (è il caso quest'ultimo del graphic novel, etichetta che spesso viene assunta "ad ombrello" per indicare indistintamente tutti i testi all'incrocio tra fumetto e narrazione verbale lunga e per «posizionare il prodotto sia in libreria sia rispetto alle aspettative dei lettori e degli addetti ai lavori $\gg^{30}$ ) è significativa del ruolo che questa forma d'arte assume come punto di vista privilegiato per indagare le dinamiche di contaminazione tra media e forme diverse ${ }^{31}$.

elettronici, Milano, Apogeo, 2007; M. Giovagnoli, Cross-Media. Le nuove narrazioni, Milano, Apogeo, 2009.

30 F. Fasiolo, Italia da fumetto. Graphic journalism e narrativa disegnata nel racconto della realtà italiana di ieri e di oggi, Roma, Tunué, 2012, 21.

31 Non è un caso che proprio all'intreccio tra letteratura e arti visive, con grande attenzione per graphic novel e fototesto, sia stato dedicato il convegno annuale MOD (Società italiana per lo studio della modernità letteraria) 2017. Cfr. R. Gasperina Geroni 
Ma per comprendere a pieno il fenomeno del graphic journalism, per come si è definito negli ultimi venticinque anni, esso va letto, all'interno della più ampia fioritura dei Visual Studies, come conseguenza della sempre più ampia permeabilità tra forme artistiche e linguaggi un tempo separati ${ }^{32}$. Nell'analisi dell'iconotesto torna utile l'accenno precedente di Giovannetti alla «vettorialità della mescolanza»: parole e immagini entrano in tensione e la fruizione dell'opera cambia a seconda del significato e dell'interazione tra la parte verbale e la parte visuale ${ }^{33}$. Il rapporto tra testo e immagine può assumere forme diverse, di maggiore o minore prossimità o integrazione: dal caso di Senza verso (2004) di Emanuele Trevi dove le immagini, qualitativamente varie (fotografie, immagini d'archivio, immagini pittoriche), non hanno un'autorialità definita e sono poste al fondo in una posizione di subordinazione alla parola scritta, a Zingari di merda (2008) di Antonio Moresco in cui le foto di Giovanni Giovannetti, benché ugualmente relegate al fondo del testo, «narrano da un altro punto di vista, quello del fotografo, la vicenda cui Moresco ha dato forma con le parole» ${ }^{34}$, fino al caso di Absolutely Nothing (2016) con cui Giorgio Vasta realizza un diario-reportage, nel quale l'autobiografia e l'autofinzione sconfinano nel territorio del resoconto di viaggio, corredato da un'appendice fotografica a firma di Ramak Fazel. Il diario fotografico di Vasta e Fazel si presenta così come un prodotto difficilmente etichettabile, un iconotesto complesso in cui il deserto nel quale i protagonisti sono collocati si configura allo stesso tempo come sfondo paesistico

e F. Milani (a cura di), La modernità letteraria e le declinazioni del visivo. Arti, cinema, fotografia e nuove tecnologie. Atti del XIX Convegno Internazionale della MOD 22-24 giugno 2017. Tomi I-II, Pisa, ETS, 2019.

32 Nell'ottica pluri- e transdisciplinare dei Visual Studies l'immagine viene indagata con gli strumenti della filosofia, della storia, della sociologia, con attenzione particolare ai nuovi media visivi e digitali, allo scopo di ricostruire e comprendere la catena di produzione-ricezione in cui è inserita. Cfr. A. Pinotti, A. Somaini, Cultura visuale. Immagini sguardi media dispositivi, Torino, Einaudi, 2016 e qui la Premessa di Clelia Martignoni.

33 Tra i molti e interessanti studi sull'iconotesto restano imprescindibile punto di partenza i saggi di Michele Cometa, in particolare: M. Cometa, Fototesti. Per una tipologia dell'iconotesto in letteratura, in V. Del Marcio, I. Pezzini (a cura di) La fotografia. Oggetto teorico e pratica sociale, Roma, Edizioni Nuova Cultura, 2011; Id. Forme e retoriche del fototesto letterario, in $\mathrm{M}$. Cometa e R. Coglitore (a cura di) Fototesti, Macerata, Quodlibet, 2016.

34 Cfr. G. Carrara, Per una fenomenologia dell'iconotesto narrativo ipercontemporaneo, in «Comparatismi» 2 (2017), 44. 
e dispositivo per far emergere l'interiorità dei suoi personaggi. In questa prospettiva Giuliana Benvenuti, esperta di temi transmediali $i^{35}$, sottolinea bene come «insieme al filone reportagista, resta poi produttivo soprattutto quello autobiografico/autofinzionale, nel quale si realizza un "nuovo patto foto-autobiografico", necessario alla peculiare narrazione fototestuale del sé» ${ }^{36}$.

L'esperienza dello spazio, che trova il suo corrispettivo in fotografia nella tecnica del ritratto ambientato, intercetta un'ulteriore interessante "contaminazione" tra saperi e metodologie, tra ecologia e letteratura, verso nuovi approcci ecocritici ${ }^{37}$ che sfruttano appunto l'ecologia come "struttura di senso". È Niccolò Scaffai, con Serenella Iovinoo, Caterina Salabè e Alberto Casadei ${ }^{40}$, ad aver portato il tema all'attenzione di italianisti e comparatisti mostrando, con sensibilità critica e civile, quanto le questioni ambientali possano essere proficuamente impiegate per rileggere oggi testi capitali della nostra letteratura, alla luce di una reciproca implicazione: «da un lato, infatti, il discorso ecologico ha adottato costruzioni narrative tipicamente letterarie, restituendole poi sotto forma di connotati caratteristici del romanzo a tema

35 Tra i suoi interventi su letteratura e altri media si vedano soprattutto i più recenti: G. Benvenuti, Il Brand Gomorra. Dal romanzo alla serie TV, Bologna, Il Mulino, 2017; Ead., Fictions, transmedialité et processus de transculturation, in «Ecritures» (2018), 109-120; Ead., Il Graphic Novel: una quaestio de centauris?, in R. Gasperina Geroni e F. Milani (a cura di), La modernità letteraria e le declinazioni del visivo, op. cit., 83-102; Ead., Nuovi realismi e fumetto di realtà, in «Narrativa», (2019), 55-65; G. Benvenuti, S. Colaone, L. Quaquarelli (a cura di), Bande à part 2. Fumetto e transmedialità, Milano, Morellini, 2018.

36 G. Benvenuti, Il ritratto ambientato tra letteratura e fotografia, in A mezzi termini, op. cit., 110.

37 Il termine "Ecocriticism" è stato coniato da William Rueckert nel 1978 (anche se la nascita dell'omonimo filone di studi risale all'inizio degli anni Novanta). Cfr. W. Rueckert, Literature and Ecology: an Experiment in Ecocriticism, in «Iowa Review», 9, 1 (1978), 71-86.

38 S. Iovino, Ecologia letteraria. Una strategia di sopravvivenza, Milano, Ambiente, 2006; Ead., Ecocriticism and Italy: Ecology, Resistance and Liberation, London-New York, Bloomsbury, 2016.

39 C. Salabè (a cura di), Ecocritica. La letteratura e la crisi del pianeta, Roma, Donzelli, 2013.

40 A. Casadei, Poetiche della creatività. Letteratura e scienze della mente, Milano, Mondadori, 2011; Id, Biologia della letteratura. Corpo, stile, storia, Milano, Il Saggiatore, 2018. 
ambientale [...] dall'altro lato, la letteratura ha trovato nell'ecologia sia argomenti originali (quello dei rifiuti, ad esempio), sia elementi per rinnovare temi classici come quello della fine del mondo» ${ }^{41}$. Attraverso lo straniamento e lo studio del punto di vista, il pensiero ecologico permette di superare la visione antropocentrica per ingaggiare una riflessione sull'io e l'ambiente che tiene conto dell'ambivalente desiderio di sintonia e separazione tra uomo e natura, delle trasformazioni del paesaggio operate dall'uomo e dei rischi a cui oggi più che mai va incontro il nostro pianeta. La contaminazione di sguardi e di approcci, quello ecologico e quello letterario, di forme e di generi, il saggio, l'aforisma, la poesia, trova un'originale esemplificazione in testi recenti come In territorio selvaggio ${ }^{42}$ di Laura Pugno, «saggio letterario», «taccuino di note», «diario in pubblico» ${ }^{43}$ che sfrutta tutta la duttilità del discorso saggistico per fondere la riflessione ecologica sull'io e sul paesaggio all'analisi critica della nostra letteratura presente.

Ciò che la mescolanza tra generi, forme e approcci mette in luce è una questione di "prospettiva". Ampliando il discorso possiamo concludere che lo sguardo ecologico, così come la fusione del verbale e del visivo, lo sconfinamento della letteratura nel giornalismo, l'utilizzo di cinema e fotografia, si esprimono nella capacità di cogliere e rappresentare relazioni, sottolinearne gli effetti inediti e dare loro forma, nel tentativo di trovare un modo, sempre parziale e in movimento, per configurare e interpretare la complessità.

41 N. Scaffai, Letteratura e ecologia, Roma, Carocci, 2017, 12-13.

42 L. Pugno, In territorio selvaggio, Milano, Nottetempo, 2018.

43 Sono tutte etichette che sono state richiamate dall'autrice o dai recensori per classificare il libro. Cfr. soprattutto In territorio selvaggio. Dialogo tra Laura Pugno e Massimiliano Manganelli, in «Nazione Indiana» 2 luglio 2019 (consultabile online al link https://www.nazioneindiana.com/2019/07/02/in-territorio-selvaggio/). 
